Original Research Paper

\title{
An Investigation of Tradeoffs in Herbicide Resistant Brassica rapa: Effects on Pollen and Ovule Production, Biomass and Development
}

\author{
${ }^{1}$ Robin Bingham, ${ }^{1}$ Pamela King and ${ }^{2}$ Andrew G. Keck \\ ${ }^{I}$ Department of Natural and Environmental Sciences, \\ ${ }^{2}$ Department of Mathematics and Computer Science, \\ Western State Colorado University, Gunnison, Colorado 81231, USA
}

Article history

Received: 18-07-2016

Revised: $10-12-2016$

Accepted: 07-01-2017

Corresponding Author: Robin Bingham

Department of Natural and

Environmental Sciences,

Western State Colorado

University, Gunnison,

Colorado 81231, USA

Tell: $970-943-3355$

Fax: 970-943-7120

Email: rbingham@western.edu

\begin{abstract}
For decades, herbicides have been used to control weeds and increase crop yields, a practice resulting in a growing number of weeds that are herbicide resistant. Like the problem of antibiotic resistance for medicine, herbicide resistant weeds present a significant agricultural challenge. To meet this challenge, we need management strategies informed by a more complete understanding of the biology of herbicide resistance. With this goal in mind we investigated tradeoffs in growth and reproduction of Atrazine Resistant (AR) versus Atrazine Susceptible (AS) Brassica rapa grown at two temperature regimes. Between the strains, tradeoffs were evident for pollen production and overall plant development. AS plants produced significantly more pollen per flower compared to AR plants. Additionally, development was significantly delayed in AR as compared to AS plants. Atrazine resistant plants took $20.5 \%$ longer than atrazine susceptible plants to produce their first true leaves, $13.0 \%$ longer to produce buds and $8 \%$ longer to produce the first flower as compared to the atrazine susceptible strain. Biomass, ovule count and pollen count were all greater in the cool treatment, however growth was significantly delayed as compared to the warm treatment. Plants in the cool treatment had significantly greater above ground biomass $(26.5 \%)$ than plants in the warm treatment, but there was no effect of strain on biomass. Similarly, we found a significant effect of temperature, but not strain, on the average number of ovules produced per flower, with the warm treatment plants producing significantly fewer ovules than plants in the cool treatment. Warm temperatures also had a significant negative effect on pollen production. This study expands our understanding of tradeoffs associated with herbicide resistance to include effects on components of both male and female fitness.
\end{abstract}

Keywords: Herbicide Resistance, Atrazine, Tradeoffs, Pollen, Brassica rapa

\section{Introduction}

For decades, herbicides have been used to control weeds and increase crop yields, a practice resulting in a growing number of weeds that are herbicide resistant. Like the problem of antibiotic resistance for medicine, herbicide resistant weeds present a significant agricultural challenge (Norsworthy et al., 2012). The first reported instance of Herbicide Resistance (HR) was in 1957 and since then cases have grown exponentially
(Hilton, 1957; Prather et al., 2000).Weeds such as rigid ryegrass (Lolium rigidum), wild oats (Avena fatua), redroot pigweed (Amaranthus retroflexus), smooth pigweed (Amaranthus hybridus), green foxtail (Setaria viridis) and horseweed (Conyza canadensis) are among the most economically significant weeds to develop HR biotypes (Beckie, 2006). In the United States, the estimated cost of corn and soybean crop loss due to weeds is over $\$ 43$ billion annually (Weed Science Society of America). Adding to the problem, current HR 
management strategies are estimated to cost the same as strategies that help prevent or delay resistance, providing no incentive for a more preemptive approach (Beckie, 2006). To meet this challenge, we need management strategies informed by a more complete understanding of the ecological and evolutionary tradeoffs associated with HR which can, in turn, contribute to the development of alternative management strategies and more effective and environmentally sensitive weed control (Norsworthy et al., 2012).

Evolutionary tradeoffs occur when traits that are genetically correlated exhibit antagonistic pleiotropy in which increased performance in one or more traits is correlated with reduced performance in others. Antagonistic pleiotropy imposes limits to adaptation, which may explain why herbicide resistant weeds have not outcompeted herbicide susceptible forms. For example, research into tradeoffs associated with herbicide resistance has shown that triazine-resistant plants are more susceptible to herbivores than triazinesusceptible plants in Amaranthus hybridus (Gassmann, 2005; Gassmann and Futuyma, 2005) and to the rust fungus pathogen in Senecio vulgaris (Salzmann et al., 2007). Resistance to triazine is also associated with a decrease in overall photosynthesis (Salzmann et al., 2007). In glyphosate-resistant Lolium rigidum, researchers found that resistant plants had lower fitness as quantified by differences in seed production, with resistant plants producing fewer (but larger) seeds compared to susceptible plants (Pedersen et al., 2006). Similarly, chlorsulfuron-resistant Arabidopsis thaliana individuals produce $26 \%$ fewer seeds than susceptible individuals (Purrington and Bergelson, 1997). Herbicide resistance has also been documented to have negative pleiotropic effects associated with seed characteristics such as germination and seedling emergence (Délye et al., 2013b).

Additionally, the magnitude of these negative effects often depends on the environment in which the plants are grown. For example, Plowman and Richards (1997) conducted a study comparing competitive ability between resistant and susceptible biotypes of Brassica rapa. Their study consisted of two light levels and three temperature treatments. They found that under high light the susceptible strain was more competitive in the high and medium temperature treatments and the resistant strain was the better competitor at the low temperature. However, under low light conditions the situation was reversed. This reversal of competitive ability between $B$. rapa's resistant and susceptible biotypes is a good example of the complexity of pleiotropic effects that are still poorly understood (Délye et al., 2013a).

The genetic mechanisms behind herbicide resistance include both monogenic and polygenic systems. To date most research on herbicide resistance has focused on monogenic systems, those in which resistance is conferred by alleles at a single gene locus either nuclear, such as resistance to herbicides that inhibit Acetolactate Synthase (ALS), or cytoplasmic, such as in resistance to triazine herbicides. This type of resistance is referred to as Target-Site-Resistance (TSR). Polygenic non-targetsite-resistance (NTSR) also occurs and has recently been recognized as the predominant mechanism of resistance against some important herbicides including glyphosate. However, research on the mechanisms behind NTSR is lacking and the genetic complexity of polygenic adaptations to herbicides remains to be elucidated (Délye et al., 2013a).

TSR typically occurs from the mutation of a single site in a plant's genome. For instance, atrazine, a broad spectrum herbicide targeted at both broadleaf and grass weeds, is part of the triazine family which kills by binding with the quinone $B\left(Q_{B}\right)$ protein in the photosystem II electron transport chain preventing photosynthetic electron transport (LeBaron et al., 2008). Resistance occurs when mutations change the $\mathrm{Q}_{\mathrm{B}}$ protein's amino acid sequence which lessens the ability of the herbicide to bind with the protein. The shift of glycine in place of serine at position 264 is the most common of these mutations (Ser-264-Gly) (Shukla and Devine, 2008). This mutation results in significantly reduced photosynthetic potential resulting in lower growth rates, reduced competitive ability and lower reproductive output as measured by seed production (Vila-Aiub et al., 2009).

As noted above, previous work on tradeoffs associated with herbicide resistance has demonstrated negative impacts on biomass, photosynthesis and seed production, as well as the associated ecological costs of greater susceptibility to herbivory and reduced competitive ability (Purrington and Bergelson, 1997; Arntz et al., 2000; Gassmann, 2005; Gassmann and Futuyma, 2005; Salzmann et al., 2007; Vila-Aiub et al., 2009; Délye et al., 2013a). However, no research that we are aware of has investigated the impact of herbicide resistance on components of male fitness, such as pollen production. Given concerns about the spread of herbicide resistance through gene flow from genetically modified crops into weedy relatives and the importance of out crossing for the evolution of NTSR (Délye et al., 2013a) it would be worth knowing to what extent gene flow through pollen might be reduced by negative pleiotropic effects on pollen production.

In this study we investigated the impact of resistance to the herbicide atrazine on pollen production as well as on growth and development of Brassica rapa. We predicted that Atrazine Resistant (AR) individuals would produce less pollen, fewer ovules, less above ground biomass and later average dates for first flower, first buds and first true leaves than Atrazine Susceptible (AS) 
plants. Because tradeoffs are often difficult to detect and have been shown to be impacted by environmental conditions, we varied the thermal conditions to introduce environmental variability.

\section{Materials and Methods}

\section{Study System}

Brassica is a genus known for its diverse crop species and significant economic value (Williams and Hill, 1986). Along with important agricultural species, the genus contains some common weeds (e.g., B. kaber, $B$. nigra, $B$. juncea). Rapid-cycling populations of Brassica rapa have been developed for use as a model organism making possible the exploration of plant development, fecundity and evolution over short periods of time and multiple $B$. rapa genotypes are available for research including those that are atrazine resistant (Williams and Hill, 1986). We used rapid-cycling B. rapa obtained from the Rapid Cycling Brassica Collection (University of Wisconsin, Department of Plant Pathology, Madison, Wisconsin) to compare the growth and development of plants that are susceptible to atrazine (AS) and those that are resistant (AR).

\section{Experimental Design}

Two hundred and four rapid-cycling B. rapa plants were grown individually in $5 \mathrm{~cm}$ pots randomly placed in growth chambers. We used 52 plants per strain (AR and AS) and two temperature treatments $\left(24^{\circ} \mathrm{C} / 19^{\circ} \mathrm{C}\right.$ and $17^{\circ} \mathrm{C} / 12^{\circ} \mathrm{C}$ ). We varied the thermal environment to introduce environmental variability, since negative pleiotropic effects are often small and may be influenced by environmental conditions. Plants received equal amounts of light and water. We monitored plants regularly to record the date at which each plant developed its first true leaves, buds and flowers. Flowers were harvested on the third day after opening. For the first four flowers of an individual plant we weighed the stamens and placed them in a centrifuge tube with 100 $\mu \mathrm{L}$ of $100 \%$ ethanol for pollen preservation. The carpels were dissected and the ovules counted. Centrifuge tubes of stamens were vortexed for approximately $30 \mathrm{sec}$, after which pollen counts were performed using a hemocytometer (Kearns and Inouye, 1993). After flowers were collected for pollen and ovule counts, we harvested the plants to quantify above ground biomass.

\section{Data Analysis}

We analyzed the data with two factor ANOVA in JMP (2016) as well as with linear models in R (R Core Team, 2016). Because the data for biomass and the development variables (1st true leaves, buds and flowers) were right skewed we used a log transformation prior to analysis to meet the assumptions of ANOVA. A cube-root transformation provided the best fit for statistical assumptions for the pollen data. The complete pollen data were used for an initial analysis. Due to a large number of plants producing zero pollen or very low numbers we also analyzed the data after removing those that produced below 200 pollen grains per flower. We believe these low numbers occurred due to contamination during the counting process and therefore conducted recounts of those samples confirming that they should be excluded as zero pollen values. To determine whether the production of flowers and pollen was nonrandom across strains and treatments we used a Fisher Exact Test for Proportions.

\section{Results}

We found a significant effect of plant strain on pollen production, but the model only explained $1.0 \%$ of the variation in pollen grain number. Many of the atrazine susceptible plants produced flowers without any pollen, but of the plants that did produce pollen the AS plants produced significantly more pollen per flower compared to the atrazine resistant strain $\left(\mathrm{r}^{2}=0.011, \mathrm{p}<0.05\right.$, Table 1). The number of plants with no flowers, flowers without pollen and flowers with pollen was nonrandom as determined by the Fisher exact test for proportions $(p<0.001)$ (Fig. 5). The effect of temperature on production of flowers with and without pollen was dependent on strain (Fig. 5 and 6). Figure 6b (number of flowers without pollen) shows strains reacting similarly across treatments; both produced fewer flowers in the warm treatment. However, Fig. 6a (total flowers) and Fig. 6c (flowers with pollen) show an interaction between strain and treatment. In the warm treatment AS plants produced more flowers than in the cold treatment, while the AR plants produced fewer (Fig. 6a). When comparing flowers with pollen between cold and warm treatments (Fig. 6c) there was a strong effect of temperature on the AS plants; AS plants produced many more flowers with pollen in the warm treatment than in the cold treatment. In contrast, there were only minor differences in the number of pollen producing flowers between cold and warm treatments for the AR plants.

There was also a significant effect of both strain and temperature on the time to produce the first true leaves, buds and flowers (Table 1, Fig. 3). Not surprisingly, plants in the cool treatment developed more slowly than the plants in the warm treatment $(33.7,27.6$ and $34.8 \%$ longer for leaves, buds and flowers respectively). In the cool treatment atrazine resistant plants took 3.2 days longer than atrazine susceptible plants to produce their first true leaves, 2.9 days longer to produce buds and 2.6 days longer to produce the first flower as compared to the atrazine susceptible strain. In the warm treatment atrazine resistant plants took 2.4 days longer than atrazine susceptible plants to produce their first true 
leaves, 2.3 days longer to produce buds and 1.9 days longer to produce the first flower as compared to the atrazine susceptible strain. Somewhat paradoxically, plants in the cool treatment had significantly greater above ground biomass $(26.5 \%)$ than plants in the warm treatment, but there was no effect of strain on biomass (Table 1, Fig. 1). Similarly, we found a significant effect of temperature, but not strain, on the average number of ovules produced per flower, with the warm treatment plants producing significantly fewer ovules than plants in the cool treatment $\left(\mathrm{r}^{2}=0.106, \mathrm{p}<0.001\right.$, Table 1 , Fig. 2). Warm temperatures also had a significant negative effect on pollen production (Table 1, Fig. 4).

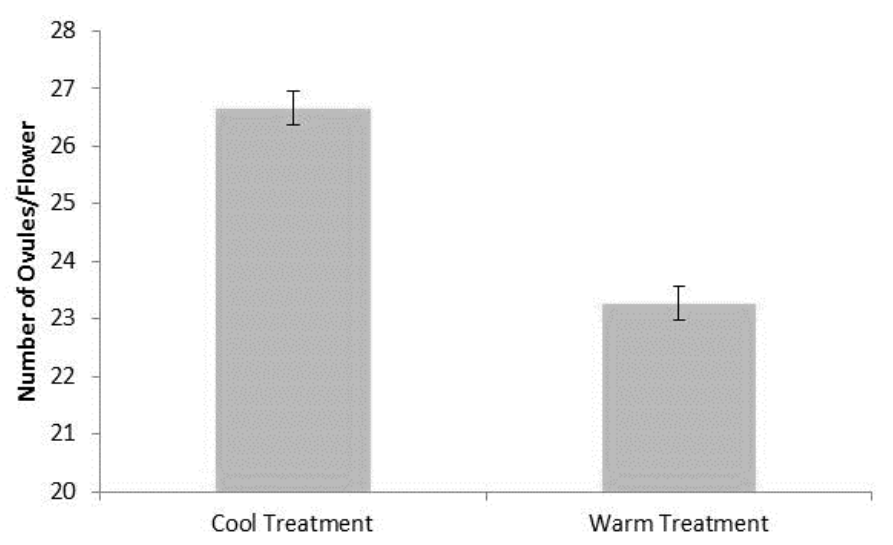

Fig. 2. Effect of temperature on mean ovule number, with standard error, $\mathrm{p}<0.001$
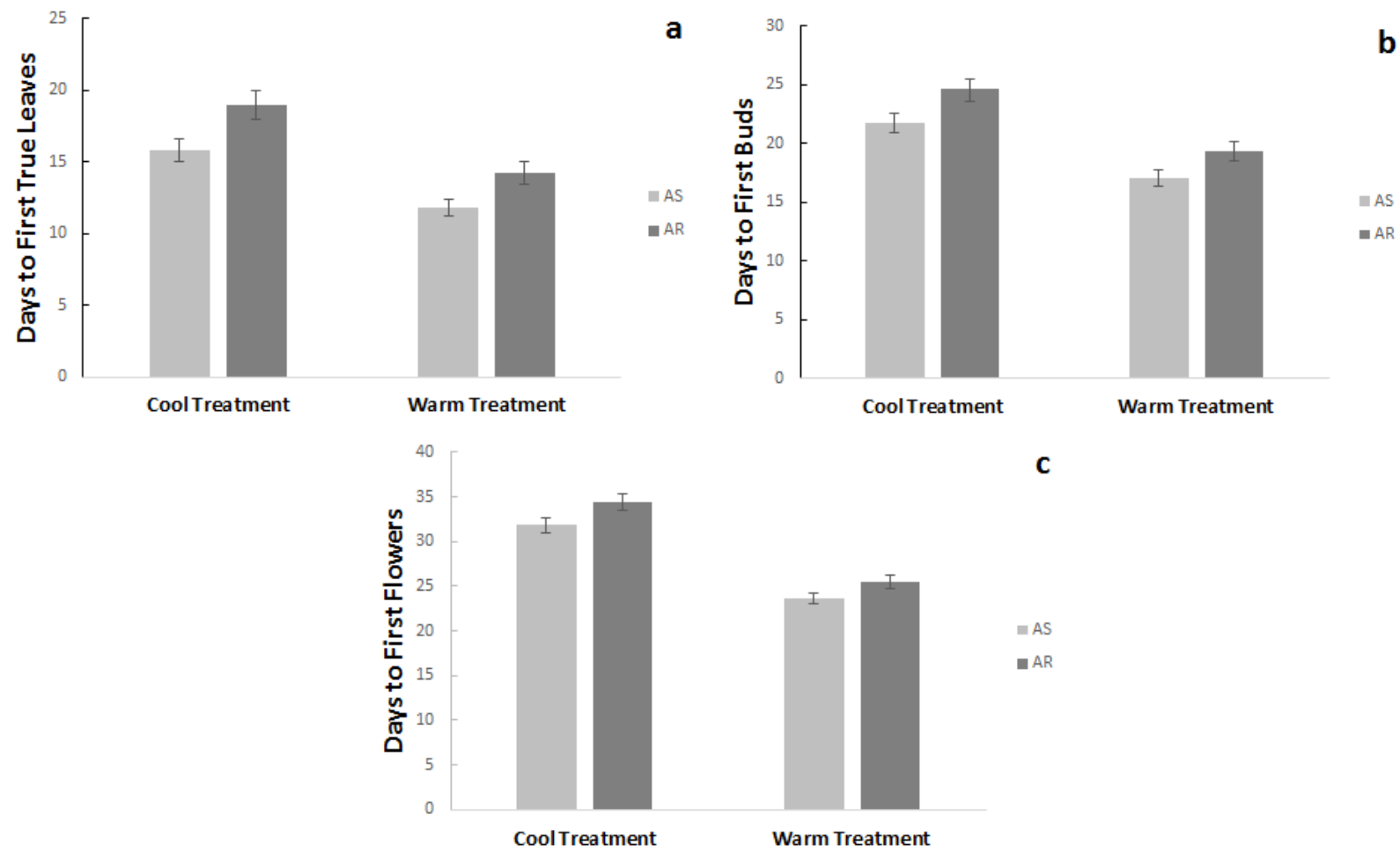

Fig. 3. Comparison of AS and AR for days to (a) first true leaves, (b) buds and (c) flowers in cool and warm treatments, with standard error bars. For significance values see Table 1

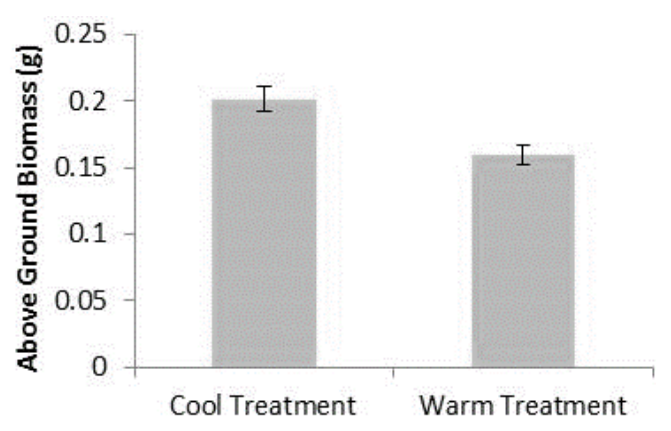

Fig. 1. Effect of temperature on mean above-ground biomass, with standard error, $p<0.001$ 


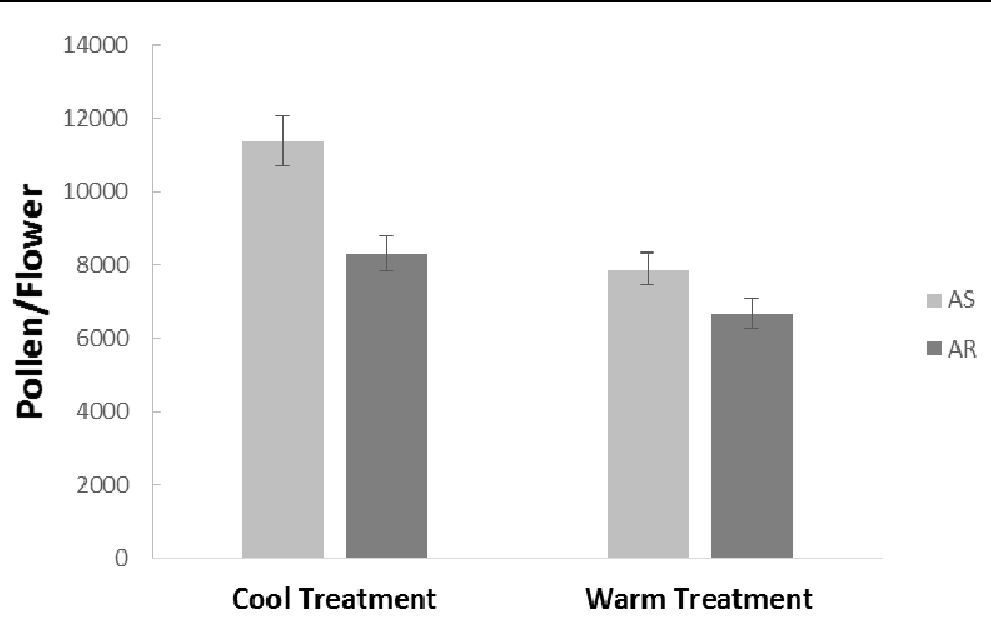

Fig. 4. Comparison of the number of pollen grains per flowers for AS and AR populations in both cool and warm treatments with standard error bars. For significance values see Table 1

Table 1. Results of the ANOVA. The data for biomass and the development variables ( $1^{\text {st }}$ true leaves, buds and flowers) were log transformed prior to analysis to meet the assumptions of ANOVA. A cube-root transformation provided the best fit for statistical assumptions for the pollen data. NS = not significant

\begin{tabular}{lllllll}
\hline & Biomass & 1st True leaves & 1st Buds & 1st Flowers & Ovules & Pollen \\
\hline Strain & NS & $\mathrm{p}<0.05$ & $\mathrm{p}<0.05$ & $\mathrm{p}<0.05$ & NS & $\mathrm{p}<0.05$ \\
Temperature & $\mathrm{p}<0.001$ & $\mathrm{p}<0.001$ & $\mathrm{p}<0.001$ & $\mathrm{p}<0.001$ & $\mathrm{p}<0.001$ & $\mathrm{p}<0.05$ \\
Strain $x$ Temperature & NS & NS & NS & NS & NS & NS \\
\hline
\end{tabular}

\section{Discussion}

Atrazine resistance results from a mutation that interferes with photosynthetic electron transport and thus, photosynthetic efficiency. Therefore, costs in the form of slower development are not surprising. As reported in other studies (Jordon, 1996; Plowman and Richards, 1997; Vila-Aiub et al., 2009), we also found this to be true. AR individuals took $20.5 \%$ longer to produce their first true leaves, $13 \%$ longer to produce buds and $8 \%$ longer to produce flowers than AS plants. These differences reflect reductions in resource acquisition that can affect competitive ability (Plowman and Richards, 1998) as well as resource allocation to defense (Gassmann, 2005; Gassmann and Futuyma, 2005).

Motivated by interest in the evolutionary consequences of herbicide resistance, several studies have investigated the effects of herbicide resistance on fitness and in many cases the predicted negative pleiotropic effects were found (Purrington and Bergelson, 1997; Vila-Aiub et al., 2009; Délye et al., 2013a). Ours is the first to report a significant negative effect of herbicide resistance on pollen production and though the proportion of variance explained by our model is small, our results suggest that costs through male function warrant further investigation across both TSR and NTSR systems. Effects on female fitness as measured by maternal traits such as seed set, or on seed characteristics themselves (germination, seed mass, etc.) have been reported in numerous previous studies (Purrington and Bergelson, 1997; Arntz et al., 2000; Salzmann et al., 2007; Vila-Aiub et al., 2009; Délye et al., 2013a). These studies mostly report significant negative effects of HR on female function, but this is not always the case (Pedersen et al., 2006). The lack of a significant effect of strain on ovule number in the present study echoes this ambiguity and underscores the challenge of accurately quantifying the costs of herbicide resistance.

Disentangling some of the complexities surrounding biological costs is possible when care is taken to control for genetic background. For example, Purrington and Bergelson (1997) created Near Isogenic Lines (NIL) of HR and HS Arabidopsis thaliana, which were grown under two levels of resource availability. As measured by seed production, their results showed significant fitness costs for NIL HR lines at both resource levels. Their experimental design allowed them to confidently conclude that the reduced fitness effects were due to the resistance gene itself (Purrington and Bergelson, 1997). Controlling for genetic background can reduce the variation in response variables making detection of fitness costs more likely as well as allowing accurate identification of the causal agent of those costs. Since we did not control for genetic background in the present study, we cannot unambiguously point to the AR mutation as the cause of the fitness costs herein reported; however, we suggest that our results are a conservative estimate of the costs of atrazine 
resistance in B. rapa within an ecologically relevant context since the resistance gene will likely be found in diverse genetic backgrounds. Moreover, even in studies for which genetic background was meticulously controlled, mixed results have been reported (Jordon, 1996).

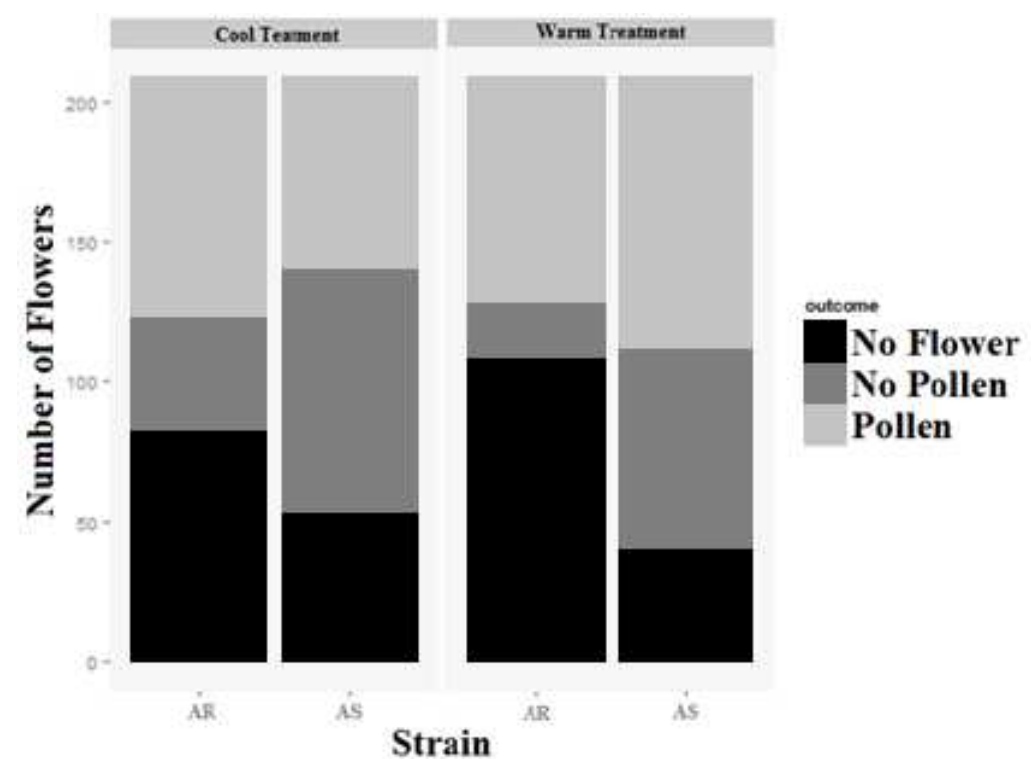

Fig. 5. The number of plants with no flowers, flowers without pollen and flowers with pollen was nonrandom as determined by the Fisher Exact Probability test $(\mathrm{p}<0.001)$
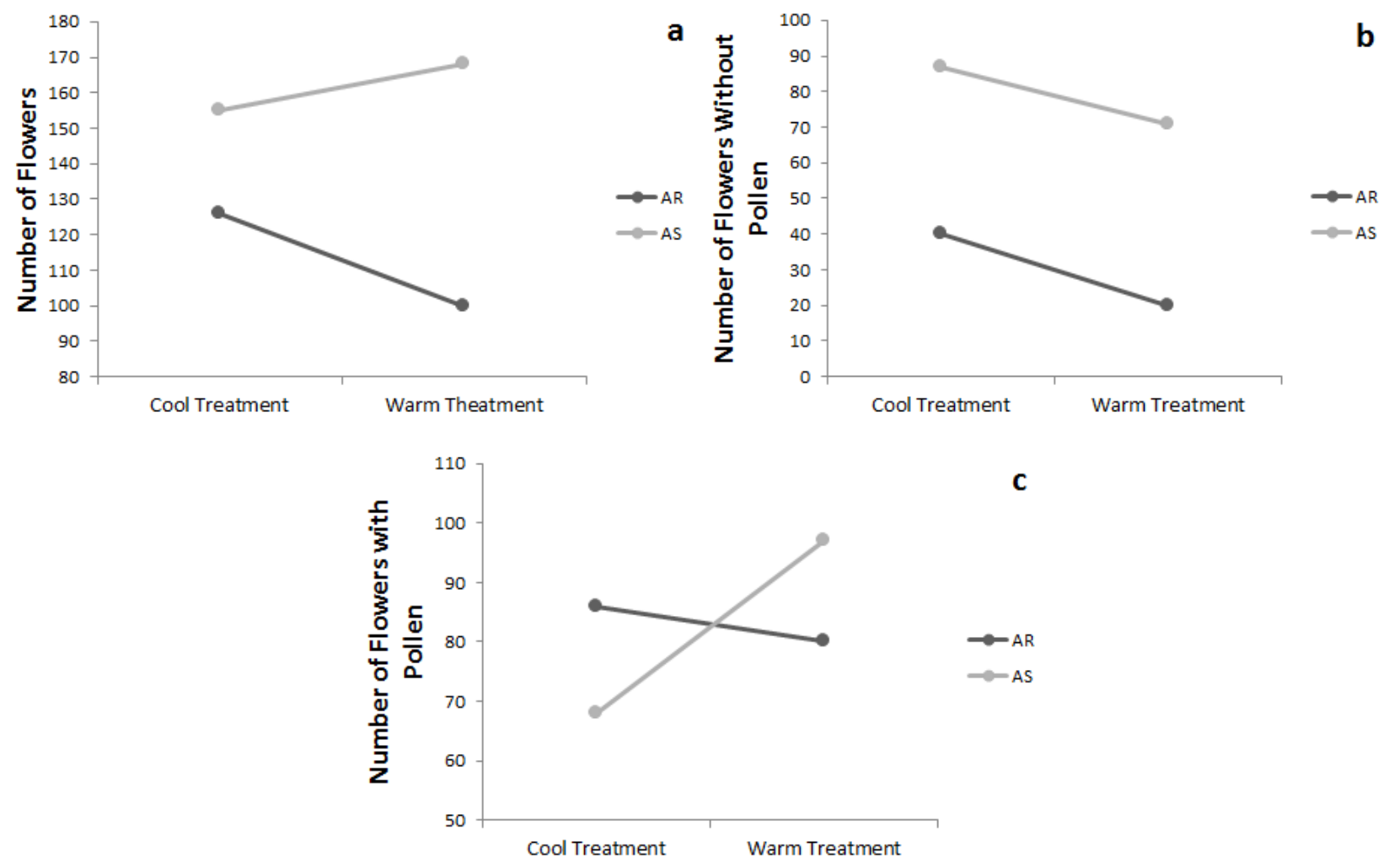

Fig. 6. Relationships between strain and temperature for (a) total flowers, (b) flowers without pollen and (c) flowers with pollen 
Contributing to the challenge of quantifying costs of resistance are the findings that they may only be evident when plants are grown under stressful conditions. In both studies referenced above (Jordon, 1996; Purrington and Bergelson, 1997), HR and HS plants were compared under conditions intended to test this hypothesis. Purrington and Bergelson grew plants under high and low resource levels and Jordan tested plants grown within different competitive regimes. Similarly, Plowman and Richards (1997) grew HR and HS $B$. rapa in a competitive replacement series under differing light and temperature regimes. Similar to Jordon (1996), they ensured that nuclear genotypes of the HR and HS strains were comparable. They found that HS was the superior competitor for all treatments, except at the regimes of high light/low temp and low light/high temp. Their study showed that slight variations of light and temperature can have great effects over who is the better competitor. Likewise, we incorporated two temperature regimes to assess costs under different environments. Similar to the findings reported by Plowman and Richards (1997), we found that strain interacted with temperature to affect both flower and pollen production (Fig. 5 and 6). Flower production in AR plants was negatively affected in the warm temperature treatment, in contrast to the AS plants which produced more flowers under warm conditions as well as more flowers with pollen (Fig. 5 and 6).

\section{Conclusion}

Understanding the ecological and evolutionary tradeoffs associated with HR can inform our approaches to weed management. Having a complete picture of fitness tradeoffs is essential to this endeavor. Although fitness costs are often quantified through seed production and biomass, the impact on pollen production has never been explored. Our results add to a growing collection of studies demonstrating biological tradeoffs associated with herbicide resistance and are the first to quantify costs through male function. It is also becoming clear that fitness tradeoffs associated with HR are dependent on environmental factors such as light, temperature and resource availability, likely among others. Further exploration of abiotic influences on both male and female fitness tradeoffs are warranted and should be considered as part of an effective weed management approach.

\section{Acknowledgement}

We thank the Thornton Biology Research Program at Western State Colorado University for financial support.

\section{Author Contributions}

P.K. and R.B. conducted all experiments and wrote the manuscript. P.K and A.K conducted statistical analyses. All authors interpreted results and commented on the manuscript.

\section{Conflict Of Interest}

None declared.

\section{References}

Arntz, M.A., D.H. DeLucia and N. Jordan, 2000. From fluorescence to fitness: Variation in photosynthetic rate affects fecundity and survivorship. Ecology, 81: 2567-2576. DOI: $10.1890 / 0012-$ 9658(2000)081[2567:FFTFVI]2.0.CO;2

Beckie, H.J., 2006. Herbicide-resistant weeds: Management tactics and practices. Weed Technol., 20: 793-814. DOI: 10.1614/WT-05-084R1.1

Délye, C., M. Jasieniuk and V. Le Corre, 2013a. Deciphering the evolution of herbicide resistance in weeds. Trends Genet., 29: 649-658. DOI: $10.1016 /$ j.tig.2013.06.001

Délye, D., Y. Menchari, S. Michel, É. Cadet and V. Le Corre, 2013b. A new insight into arable weed adaptive evolution: mutations endowing herbicide resistance also affect germination dynamics and seedling emergence. Annals Botany, 111: 681-691. DOI: $10.1093 / \mathrm{aob} / \mathrm{mct} 018$

Gassmann, A.J., 2005. Resistance to herbicide and susceptibility to herbivores: Environmental variation in the magnitude of an ecological trade-off. Oecologia, 145: 575-585. DOI: $10.1007 / \mathrm{s} 00442-005-0112-6$

Gassmann, A.J. and D.J Futuyma, 2005. Consequence of herbivory for the fitness cost of herbicide resistance: Photosynthetic variation in the context of plantherbivore interactions. J. Evolut. Biol., 18: 447-454. DOI: $10.1111 /$ j.1420-9101.2004.00819.x

Hilton, H.W., 1957. Herbicide Tolerant Strains of Weeds. In: Hawaiian Sugar Planters Association Annual Report, HSPA (Eds.), Hawaiian Sugar Planters Association, pp: 69-72.

Jordon, N., 1996. Effects of the triazine-resistance mutation on fitness in Amaranthus hybridus (smooth pigweed). J. Applied Ecol., 33: 141-150. DOI: $10.2307 / 2405023$

JMP, 2016. Version 12. SAS Institute Inc., Cary, NC, 1989-2016

Kearns, C.A. and D.W. Inouye, 1993. Techniques for Pollination Biologists. 1st Edn., University Press of Colorado, Niwot, ISBN-10: 0870812815, pp: 583. 
LeBaron, H.M., J.E. McFarland and O.C. Burnside, 2008. The Triazine Herbicides: A Milestone in the Development of Weed Control Technology. In: The Triazine Herbicides: 50 Years of Revolutionizing Agriculture, LeBaron, H.M., J.E. McFarland and O.C. Burnside (Eds.), Elsevier, p: 1-12.

Norsworthy, J.K, S.M. Ward, D.R. Shaw, R.S. Llewellyn and R.L. Nichols et al., 2012. Reducing the risks of herbicide resistance: Best management practices and recommendations. Weed Sci., 60: 31-62.

DOI: 10.1614/WS-D-11-00155.1

Plowman, A.B. and A.J. Richards, 1997. The effect of light and temperature on competition between atrazine susceptible and resistant Brassica rapa. Annals Botany, 80: 583-590.

DOI: $10.1006 /$ anbo.1997.0496

Pedersen, B.P., P. Neve, C. Andreasen and S.B. Powles, 2006. Ecological fitness of a glyphosate-resistant Lolium rigidum population: Growth and seed production along a competition gradient. Basic Applied Ecol., 8: 258-268. DOI: $10.1016 /$ j.baae.2006.01.002

Prather, T.S., J.M. Ditomaso and J.S. Holt, 2000. Herbicide resistance: Definition and management strategies. ANR University of California, Division of Agriculture and Natural Resources. Publication 8012.
Purrington, C.B. and J. Bergelson, 1997. Fitness consequences of genetically engineered herbicide and antibiotic resistance in Arabidopsis thaliana. Genetics, 145: 807-814. PMID: 9055089

R Core Team, 2016. R: A language and environment for statistical computing. R Foundation for Statistical Computing, Vienna, Austria.

Salzmann, D., R.J. Handley and H. Muller-Scharer, 2007. Functional significance of triazine-herbicide resistance in defence of Senecio vulgaris against a rust fungus. Basic Applied Ecol., 9: 577-587. DOI: $10.1016 /$ j.baae.2007.10.001

Shukla, A. and M.D. Devine, 2008. Basis of Crop Selectivity and Weed Resistance to Triazine Herbicides. In: The Triazine Herbicides: 50 Years of Revolutionizing Agriculture, LeBaron, H.M., J.E. McFarland and O.C. Burnside (Eds.), Elsevier, pp: 111-118.

Weed Science Society of America. http://wssa.net/wssa/weed/croploss

Williams, P.H. and C.B. Hill, 1986. Rapid-cycling populations of Brassica. Science, 232: 1385-1389. DOI: $10.1126 /$ science. 232.4756 .1385

Vila-Aiub, M.M., P. Neve and S.B. Powels, 2009. Fitness costs associated with evolved herbicide resistance alleles in plants. New Phytologist., 184:751-767. DOI: $10.1111 / \mathrm{j} .1469-8137.2009 .03055 . \mathrm{x}$ 